\title{
Un complexe de Galilée ? Penser la scène avec la science-fiction
}

Colin Pahlisch

\section{(2) OpenEdition}

1 Journals

Édition électronique

URL : http://journals.openedition.org/edl/1088

DOI : $10.4000 /$ edl. 1088

ISSN : 2296-5084

Éditeur

Université de Lausanne

\section{Édition imprimée}

Date de publication : 15 mars 2018

Pagination : 113-128

ISBN : 978-2-940331-67-3

ISSN : 0014-2026

\section{Référence électronique}

Colin Pahlisch, «Un complexe de Galilée ? Penser la scène avec la science-fiction », Études de lettres [En ligne], 1 | 2018, mis en ligne le 15 mars 2020, consulté le 11 décembre 2020. URL : http:// journals.openedition.org/edl/1088; DOI : https://doi.org/10.4000/edl.1088 


\section{UN COMPLEXE DE GALILÉE? PENSER LA SCÈNE AVEC LA SCIENCE-FICTION}

Hormis quelques rares exemples, l'étude des rapports entre théâtre et science-fiction peine encore à susciter des vocations, tant chez les chercheurs en philosophie que chez les critiques du théâtre et les dramaturges. Ce désintérêt est regrettable et cette contribution tente d'y remédier en proposant quelques approches croisées entre ce genre littéraire et l'art de la scène. À partir du concept brechtien de distanciation, il s'agira de mettre tout d'abord au jour le potentiel critique qui unit, dès l'origine, poétique de la science-fiction et théorie du théâtre. Dans un second temps, sera abordée la question de la réalisation scénique qui semble avoir souvent court-circuité la rencontre entre théâtre et science-fiction, à partir des remarques de Ralph Willingham sur diverses stratégies permettant de pallier l'impossibilité de représenter sur scène la totalité d'un monde autre, d'y faire adhérer l'imaginaire du spectateur. Enfin, le recours à la notion de sublime permettra d'embrasser d'un même regard la pensée du théâtre et la sciencefiction, et, peut-être, de jeter les bases d'une approche philosophique commune.

Origine de la philosophie, le sentiment d'étonnement, disait Platon, voilà la vraie marque du philosophe ${ }^{1}$.

Peu de travaux ont abordé spécifiquement, sous un angle philosophique, la question des rapports entre théâtre et science-fiction. Cette lacune scientifique tient sans doute autant à l'indifférence des philosophes et des spécialistes de la scène pour un genre littéraire longtemps considéré comme "mineur", ou trop "populaire", qui ne les a guère incité à tresser des liens avec leur objet d'étude. De façon similaire, on ne trouve aucune considération sur le théâtre chez Guy Lardreau, l'un des premiers

I. G. Bouchard, «Science-fiction, utopie et philosophie», p. 43. 
philosophes à s'être intéressé à la science-fiction ${ }^{2}$. La scène ne semble pas avoir de place propre au sein de l'exploration intellectuelle que mène l'auteur, pourtant tracée au cordeau de la théorie leibnizienne des mondes possibles. À croire que les codes et le potentiel artistique propres au drame ne s'avèrent pas utiles à prendre en compte dans cet exercice spéculatif qui consiste à élucider "[ce] qui, de la science, est ainsi mis en fiction ${ }^{3}$.

En ce qui concerne la recherche dramatique, on peine à trouver des sources scientifiques consacrées à l'étude de la science-fiction au théâtre, exception faite du livre de Ralph Willingham intitulé Science Fiction and the Theatre . La dualité du titre révèle d'ailleurs la césure qui a peutêtre longtemps empêché, dans l'esprit du chercheur, la réunion des deux registres de création. L'œuvre de Willingham s'en tient cependant, pour l'essentiel, à l'aspect historique des rapports entre œuvres de théâtre et de science-fiction et n'aborde que superficiellement les enjeux philosophiques que ces rapports suscitent. L'ouvrage se veut davantage un outil à destination des professionnels de la scène, visant à les convaincre de porter devant le public les pièces restées à l'état embryonnaires dans les imaginaires d'auteurs, ou celles qui attendent dans les bibliothèques des théâtres le bon vouloir de metteurs en scène audacieux.

Ainsi l'étude des liens entre science-fiction et théâtre pâtit d'un manque cruel d'appui. On sait que la philosophie ne naît pas ex nihilo, qu'il lui faut des soutiens pour s'élever des fondations pour croître. Cette contribution espère montrer que de telles fondations existent, que science-fiction et pensée de la scène ont, si ce n'est à s'entendre, du moins à converser. Il s'agit en somme de cesser d'agir comme les détracteurs du Galilée de Bertolt Brecht se refusant à coller un œil à la lunette du savant pour ne pas bouleverser leur vision du monde. Car il y a quelque chose à découvrir pour le théâtre et la philosophie dans le domaine de la sciencefiction. Si ce genre littéraire apparaît davantage abordé par les philosophes, ceux-ci rechignent trop souvent encore à prendre en compte dans leurs réflexions esthétiques la puissance propre à la scène et à ses codes. Je tâcherai donc, en premier lieu, de tisser des liens entre réflexion sur le théâtre et poétique de la science-fiction. Je tenterai ensuite de mettre

2. G. Lardreau, Fictions philosophiques et science-fiction.

3. Ibid., p. 15.

4. R. Willingham, Science Fiction and the Theatre. 
en lumière le problème central auquel se trouve confrontée l'intégration de la science-fiction au théâtre: celui de la représentation scénique. Ce dilemme constitue l'un des enjeux nodaux de l'ouvrage de Willingham cité plus haut, et la deuxième partie s'y référera essentiellement. Enfin, mon propos s'orientera vers une possible exploration philosophique des rapports entre théâtre et science-fiction, en s'appuyant sur un exemple dramatique spécifique, La Savane (The Veldt), de Ray Bradbury (1950).

\section{Une certaine lecture de Brecht}

De prime abord, on ne songerait peut-être pas à l'œuvre de Bertolt Brecht pour illustrer le fonctionnement narratif de la science-fiction. Pourtant, s'agissant d'élucider les mécanismes propres à ce type de récits, c'est bien à l'auteur de Mère Courage et ses enfants que se réfère l'un des premiers penseurs de la littérature à s'être intéressé au genre. Suivant Darko Suvin, poéticien croate, la science-fiction procède par "distanciation cognitive», c'est-à-dire qu'elle construit, par extrapolation à partir du présent de lecture, des mondes fictionnels cohérents. Une telle immersion dans un environnement imaginaire reconnaissable mais non identique au contexte d'origine de production du texte vise, dans un premier temps, à décontenancer le lecteur, à désamorcer ses habitudes. Ce procédé l'enjoint de questionner le regard qu'il porte sur le monde.

Brecht voulait écrire des "pièces pour une ère scientifique"; alors qu'il travaillait à une pièce sur Galilée, le prototype du savant, il a défini cette attitude (Verfremdungseffekt) dans son Petit Organon pour le théâtre (1948) comme une «reproduction qui permet certes de reconnaitre l'objet reproduit, mais en même temps, le rend insolite» [...]. En science-fiction, cet effet de distanciation - utilisé par Brecht différemment, dans un contexte à tendance «réaliste» - est devenu le cadre formel du genre 5 .

L'invention conceptuelle de Brecht constitue ainsi l'un des piliers fondamentaux sur lesquels s'est élaborée la théorie littéraire de la sciencefiction. L'œuvre écrite, qu'il s'agisse d'une pièce ou d'un récit, s'apparente en ce sens à un outil de pensée: le texte vient ébranler, bouleverser,

5. D. Suvin, Pour une poétique de la science-fiction, p. 14. 
"désencrasser ${ }^{6}$ dira Suvin, notre appréhension du réel. On aurait tort cependant d'attribuer à la "distanciation", qui caractérise tant les œuvres du dramaturge allemand que les textes affiliés au genre S.-F., une fonction passive. Plus qu'herméneutique, le questionnement auquel ce processus donne lieu remplit un rôle heuristique. En créant ainsi un «reflet» conjecturé de notre monde, la fiction nous enjoint à une comparaison. Lecteurs ou spectateurs sont appelés à critiquer le monde de référence duquel le texte tire sa vraisemblance. "Le miroir est un creuset» ${ }^{7}$, résume Suvin.

Une précision apparaît ici nécessaire au regard de nos propres habitudes de lecture. La science-fiction est souvent perçue comme une tentative littéraire de décryptage du futur. Au royaume des registres poétiques, elle serait investie d'un pouvoir de divination. Si une telle prétention contribue à façonner de certains auteurs une image d'augure, d'Asimov à Houellebecq, et peut s'avérer féconde en termes d'analyses posturales, elle masque cependant le véritable but des textes de sciencefiction. Ces textes contribuent, nous l'avons vu, à composer un portrait déformé, une anamorphose pourrait-on dire, du contexte d'écriture. Plus particulièrement, précise Marc Atallah, la science-fiction vise à «réfléchir à ce qu'est l'homme lorsqu'il est en permanence pétri et informé par un monde techno-industriel ${ }^{8}$. C'est donc bien à questionner certains aspects du présent que s'attachent les œuvres de science-fiction, et nullement à prédire le futur :

En l'occurrence, l'acte de déformer notre monde [qu'opère la sciencefiction] sert à mettre en évidence certaines de ses composantes, de ses défauts et de ses paradoxes en regard de la vie humaine (ce que nous percevons de notre visage dans un miroir grossissant ne se voit pas dans un miroir normal). On comprend alors mieux pourquoi cette mise en évidence n'est jamais orientée vers l'avenir mais toujours vers le présent: elle se dirige en effet vers celui qui se mire dans l'image déformée, c'est-à-dire le lecteur ou le spectateur ${ }^{9}$.

6. D. Suvin, Pour une poétique de la science-fiction, p. 6.

7. Ibid., p. 13.

8. M. Atallah, L'art de la science-fiction, p. 31.

9. Ibid., p. 40 (je souligne). 
Cet acte de "mise en évidence" par le texte de certains aspects problématiques du présent alimente, cela va sans dire, la capacité critique du lecteur. Une telle "déformation" constitue aussi un acte de création. Ce procédé poétique participe d'une volonté. Il ne s'agit pas seulement de décrire certains aspects du monde, mais aussi de chercher à le transformer. Suvin souligne en effet que la science-fiction relève d'" un procédé créateur tendant à une transformation dynamique, plutôt qu'à un simple reflet de l'environnement de l'auteur ${ }^{10}$. Une telle insistance à dépasser le constatif vers le performatif en art, trouve également dans la théorie du théâtre brechtien de frappants échos. Brecht comparait le jeu de l'acteur à une joute verbale à intention politique:

Le point de vue que le comédien adopte est un point de vue de critique sociale. Dans son élaboration des processus et sa caractérisation du personnage, il met particulièrement en avant les faits et les traits qui ressortissent au domaine social. Son jeu se transforme ainsi en un entretien avec le public sur l'état de la société $\left[. . .{ }^{11}\right.$.

Le comédien apparaît chez Brecht comme le paramètre du drame le plus susceptible de générer une prise de conscience par le spectateur en montrant le caractère malléable des règles qui structurent l'agir social et peut-être de convaincre au bout du compte les individus de leur potentiel participatif à l'égard de la société. La science-fiction, dans l'optique de Suvin, emprunte ainsi à la théorie du théâtre brechtien une certaine stimulation pour la res publica. Ces deux formes poétiques expérimentent le lien social, questionnent l'implication politique des individus, au travers de l'œuvre d'art. L'auteur du Petit organon pour le théâtre souhaite que la réalisation scénique de son écriture dramatique soit celle d'un théâtre exploratoire, visant à «fournir d'autres reproductions de la vie en commun des hommes, non seulement des reproductions d'une autre vie en commun, mais aussi des reproductions d'une autre sorte " ${ }^{12}$. De même, Suvin évoque la recherche de perfectibilité des états intersubjectifs à laquelle se rattachent les récits de science-fiction:

Ainsi, ce n'est pas seulement l'inaliénable curiosité humaine et humanisante qui donne naissance à la science-fiction. Au-delà de la

IO. D. Suvin, Pour une poétique de la science-fiction, p. 17.

II. B. Brecht, Nouvelle technique d'art dramatique, p. 337.

I2. B. Brecht, Petit organon pour le théâtre, p. 16. 
recherche fantaisiste, du jeu sémantique sans référent défini, ce genre a toujours été lié à l'espoir de trouver dans l'inconnu l'environnement idéal, la tribu, l'Etat, l'intelligence qui représente le bien suprême (ou la crainte et l'horreur de n'y trouver que son contraire) ${ }^{13}$.

Cette stimulation politique opérée par le récit révèle l'influence de l'utopie sur le genre de la science-fiction, car l'utopie procède toujours d'un élan de critique à l'égard de la société. L'historien Alain Touraine conçoit ainsi la part essentielle d'attention immanente à autrui que recèle de cette forme littéraire particulière:

L'utopie est la mise en forme de l'idée que l'être humain est entièrement social, qu'il n’a de réalité ni surnaturelle, ni individuelle [...]. L’utopie est un plaidoyer pour une société créatrice d'elle-même ${ }^{14}$.

L'utopie serait donc, selon cette définition, un élan ou un exercice mental, plutôt qu'un appel à une réalisation concrète. Nous retrouvons bien là l'idée d'un "laboratoire», d'une exploration cognitive, auxquels participent tout autant la science-fiction que le théâtre de Brecht. Ainsi la science-fiction, selon une optique suvinienne, trouve avec les considérations philosophiques de Brecht sur le théâtre de fortes connivences. La critique sociale et la visée performative à laquelle les deux théoriciens associent la création artistique peuvent sans peine servir à notre propos de premiers jalons, dans le tissage d'un horizon de recherche commun.

\section{Le problème de la représentation}

Dans un ouvrage précieux, tant par son propos que par sa rareté au sein des études académiques, intitulé Science Fiction and the Theatre, Willingham tente de comprendre la frilosité des professionnels du milieu théâtral à porter en scène des textes se revendiquant du genre. La première raison tiendrait aux préjugés nourris par ceux-ci à l'égard de la science-fiction:

13. D. Suvin, Pour une poétique de la science-fiction, p. 13.

I4. A. Touraine, "La société comme utopie», p. 28. 
Une explication consiste à dire que les artistes, tout comme les non initiés, ont tendance à tenir la science-fiction pour une littérature d'évasion [...] Pour les lecteurs non initiés, la science-fiction équivaut à un divertissement puéril ${ }^{15}$.

Aux a priori qui incitent au rejet viennent s'ajouter l'obstacle des moyens à convoquer pour contenir le monde conjecturé dans l'espace de la salle de théâtre, et convaincre le spectateur de sa vraisemblance. Selon l'auteur, un mythe tenace grève, en effet, la volonté de représenter sur scène ces extrapolations collectives qui caractérisent les pièces de sciencefiction: la nécessité d'inclure au drame des effets visuels faramineux, "c'est-à-dire que les pièces de science-fiction doivent contenir des effets spéciaux " ${ }^{16}$. Afin de déjouer cet écueil, dont le coût exorbitant sonne bien souvent le glas du projet, Willingham plaide en faveur de ce qu'il nomme un «retour à Aristote».

Dans sa Poétique [...] Aristote met perpétuellement en garde l'aspirant dramaturge contre une trop grande dépendance envers les «moyens spectaculaires» pour susciter l'émotion chez le public ${ }^{17}$.

Le succès de la science-fiction au théâtre tiendrait donc à un renouvellement de confiance envers l'imagination du spectateur:

C'est la réaction du public, et non la technologie, qui est la plus à même d'amener sur scène les mondes fantastiques de la science-fiction $^{18}$.

L'enquête menée par le critique américain fait ensuite état de quatre méthodes éprouvées par divers metteurs en scène au fil du XX $\mathrm{X}^{\mathrm{e}}$ siècle

I5. "One possibility is that theatre artists, like most uninitiated people, tend to lump all science fiction into a general category of escapist literature [...] Science fiction, to the uninitiated reader, is juvenile entertainment" (R. Willingham, Science Fiction and the Theatre, p. 4, ma traduction).

I6. "[...] that is, science fiction dramas must include special effects» (Ibid., p. 36, ma traduction).

17. «In his Poetics [...], Aristotle repeatedly cautioned the would-be dramatist against over-reliance on "spectacular means" to arouse an emotional response in the audience» (Ibid., p. 39, ma traduction).

I8. "It is audience reaction, not technology, that can bring the fantastic worlds of science fiction to the stage» (Ibid., p. 38, ma traduction). 
pour déjouer l'appétit du public envers le divertissement passif et tape à l'œil, en faisant appel à ses capacités intellectuelles et à son plaisir de reconstruire de manière simulée un monde neuf et vraisemblable. Ces méthodes ont en commun de puiser leur efficacité dans un élément proprement philosophique du rapport de l'individu au monde, ce qui sera explicité en troisième partie.

La première méthode recensée par l'auteur de Science Fiction and the Theatre pour pallier l'absence d'artifices visuels et contribuer à exciter l'imaginaire du spectateur est celle de "l'échantillonnage» (a token dose). À l'instar de la métonymie dans un texte littéraire, l'échantillonnage consiste à faire apparaître sur scène une portion des éléments dénotatifs du monde conjecturé, appelant les spectateurs à se représenter le tout. Willingham rapporte qualin de simuler une révolte mondiale de ses robots, il a suffi à Karel Čapek, auteur de R.U.R. Rossum Universal Robot (1920), de faire intervenir sur scène au terme de la pièce quelques acteurs en costume figurant ces êtres factices, et de laisser «les dialogues et l'imagination faire le reste» ${ }^{19}$.

La deuxième méthode tire précisément profit de la «distanciation cognitive» (cognitive estrangement) déjà évoquée. Elle consiste à suggérer dans le texte, et par de subtiles variations d'attitudes chez les personnages, le décalage existant dans le drame entre le monde de référence du spectateur et le monde imaginaire représenté sur scène, supposément soumis à des coutumes et des lois différentes mais rendues vraisemblables par la conjecture. Parmi d'autres exemples, Willingham cite la pièce $A P I$ 2967 de Robert Gurik (1965). Lauteur canadien nous plonge dans une société dystopique, administrée et règlementée dans les moindres détails: le plus petit paramètre de l'agir collectif est soumis au contrôle d'un pouvoir central, du taux de natalité à la fréquence des rapports sexuels et à la fonction socio-économique des individus qui la peuplent. Toute végétation a disparu depuis des siècles, or, un jour, une pomme fait son irruption dans ce monde aseptisé. L'attitude des protagonistes, empreinte d'appréhension et de stupéfaction envers cet élément organique d'un autre âge, d'apparence extrêmement banal aux yeux du spectateur, contribue à traduire l'étrangeté de l'ensemble du monde fictionnel auquel celui-ci se trouve confronté. Ce décalage ludique enjoint la

19. R. Willingham, "Dialogue and imagination do the rest» (Science Fiction and the Theatre, p. 42, ma traduction). 
capacité imaginative du spectateur, sans renforts visuels grandiloquents, à participer à la construction de la fiction à laquelle il assiste.

Mettant à profit le pôle «technique» de l'art théâtral, la troisième méthode suggérée par le critique américain propose de s'appuyer sur un "décor évocateur» (evocative setting). Nouvelle forme de métonymie, il s'agit ici de reproduire sur scène à moindres frais une parcelle matérielle valant pour un tout, de l'environnement extrapolé, afin d'appeler le spectateur à "compléter" ce dernier, à donner corps à l'illusion au plan global. Dans la pièce de l'auteur et compositeur Philip Glass intitulée 1000 Airplanes on the Roof (1988) par exemple, le voyage spatial du personnage principal, plusieurs fois kidnappé par des extraterrestres, est traduite par la projection vidéo de figures géométriques en concaténation. La répétition de celles-ci aux côtés du plateau sur deux écrans parallèles, associée à la musique de Glass, imite une profondeur de champ mouvante et kaléidoscopique, simulation du trajet irréel du protagoniste à travers nébuleuses et galaxies. Ainsi constitué, ce morceau d'espace interstellaire ouvre au spectateur une porte artificielle vers l'infini, dans laquelle son imaginaire peut s'engouffrer.

Ultime méthode relevée par l'auteur de Science Fiction and the Theatre, "le recours à l'humour" visant à faire accepter le caractère extraordinaire de l'action en invitant le spectateur à assouplir son esprit critique, à désamorcer l'attente d'effets pour laisser place à l'histoire que relate le drame et au rêve auquel celle-ci invite. Willingham rapporte, entre autres exemples, que l'adaptation scénique du long-métrage Star Wars, lors de la Convention de science-fiction Windycon IV, tenue à Chicago en 1977, incluait la présence de figurants traversant le plateau munis d'un petit écriteau où était inscrit "Effets spéciaux» au moment des scènes d'action, provoquant les rires du public ${ }^{20}$.

Ces diverses techniques restent supplétives. Elles concernent directement notre propos en ceci qu'elles signalent l'entrave principale à laquelle se trouve confrontée la volonté de penser ensemble théâtre et science-fiction: l'impossibilité de faire l'impasse sur les questions de mise en scène. Une telle contrainte s'impose sans doute à toute philosophie de l'art. Si puissants que soient les concepts esthétiques forgés par l'esprit, ils ne peuvent se permettre d'esquiver les questions pratiques et matérielles qui s'imposent directement à leur objet. Dans le cas qui nous occupe, le

20. R. Willingham, Science Fiction and the Theatre, p. 37. 
défi n'est cependant pas perdu d'avance. L'ouvrage de Willingham est truffé d'encouragements envers les professionnels du théâtre. L'auteur insiste sur le potentiel dramatique du genre science-fictionnel et n'a de cesse d'inciter les auteurs de talent à prendre la plume, car «seule une écriture dramatique de qualité est susceptible de concilier théâtre et science-fiction ${ }^{21}$.

\section{Reproduire le sublime?}

Quel serait alors l'apport proprement philosophique de la science-fiction au théâtre? La question est entière et de multiples pistes restent à tracer. Notre hypothèse est que la représentation sur scène de mondes conjecturés sur la base d'une extrapolation technoscientifique permet d'appréhender au théâtre, selon de nouvelles modalités, l'expérience esthétique et cognitive du sublime. Les divers moyens mentionnés pour attiser l'imagination du lecteur, en effet, nous semblent converger en ceci qu'ils supposent l'ouverture d'une béance ou d'une tension réflexive chez le spectateur entre l'action à laquelle il assiste et les règles étranges, non familières, du monde élargi dont cette action participe. Le sentiment de vertige auquel cet effort renouvelé de reconfiguration mentale et sensitive invite celui ou celle qui assiste au drame tient à l'immensité du champ de questionnements, à l'ampleur des conséquences sociales, techniques, économiques que laissent entrevoir au spectateur l'immersion dans le monde fictionnel conjecturé sur scène. L'expérience du drame de sciencefiction repose sur une forme radicale d'étonnement à l'égard de notre monde, autant que sur la transmission d'une certaine crainte ou d'un effroi factice. C'est ainsi qu'Edmund Burke décrivait le sublime, dans son traité de 1757. Le philosophe écossais y voyait même la plus haute émotion que l'intériorité humaine puisse appréhender:

Tout ce qui est propre à exciter les idées de la douleur et du danger; c'est à dire tout ce qui est en quelque sorte terrible, tout ce qui traite d'objets terribles, tout ce qui agit d'une manière analogue à la terreur,

2I. "[...] the only link missing between theatre and science fiction is good dramaturgy» (R. Willingham, Science Fiction and the Theatre, p. 6, ma traduction). 
est une source du sublime, ou, si l'on veut, peut susciter la plus forte émotion que l'âme soit capable de sentir ${ }^{22}$.

Burke trouve également les conséquences de cette émotion dans l'étonnement:

La passion causée par le grand et le sublime dans la nature, lorsque ces causes agissent le plus puissamment est l'étonnement, et l'étonnement est cet état de l'âme dans lequel tous ces mouvements sont suspendus par quelque degré d'horreur ${ }^{23}$.

La possibilité de rapports pertinents entre la science-fiction et l'expérience du sublime a été quant à elle abordée par Gilbert Hottois, dans son introduction à l'une des dernières études portant sur les liens du genre à la pensée. Le philosophe des sciences concède que les technosciences dans lesquelles puise l'imaginaire de la science-fiction peuvent être interprétées sous l'angle d'une relation transcendante. Le sublime qu'elles permettent de concevoir tiendrait alors à leur opérativité supposée sur le monde:

Le sublime est ce sentiment incomparable qui transporte l'être humain par l'effroi, l'émerveillement, la terreur, l'admiration... Il n'aurait pas disparu, mais il se serait métamorphosé [...]. À l'idée du sublime, les technosciences offriraient les moyens opératoires de sa réalisation. Et la transcendance devenue opératoire dans les mains de l'homme susciterait l'émotion d'un sublime renouvelé et radicalement ambivalent de l'effroi absolu qui anéantit à l'enthousiasme infini qui rapproche des dieux ${ }^{24}$.

Notons que Hottois conserve ici le mode conditionnel. C'est bien au fantasme de la réalisation de cette transcendance dans le monde qu'appartient la science-fiction. De même, c'est à une simulation de l'expérience du sublime, mais de fait, à la possibilité de sa reproduction par le spectacle, que concoure l'usage des codes de la science-fiction au théâtre. Sans doute la possibilité d'une telle simulation de l'expérience du

22. E. Burke, Recherche philosophique sur l'origine de nos idées du sublime et du beau, p. 69.

23. Ibid., p. 101.

24. G. Hottois, Science-fiction et philosophie, p. 11 (je souligne). 
sublime chez le spectateur trouve-t-elle également son explication, à un niveau anthropologique, dans le sentiment de pouvoir, le frisson d'hybris, que la technologie confère à l'homme sur le monde. L'hypothèse d'un châtiment latent et prométhéen, d'un retournement de ce pouvoir au centuple contre ceux qui l'exercent, nous semble prédisposer le spectateur à l'effroi et à l'étonnement qu'évoque le philosophe écossais. Dans cette optique, la science-fiction révèle une dimension moraliste, mais non forcément moralisante.

Une telle reproduction/simulation du sublime s'avère illustrée de façon particulièrement vive, parmi d'autres textes sans doute, chez Ray Bradbury. Dans La Savane (The Veldt, 1950), l'auteur américain met en scène une famille lambda dans un "futur proche", selon l'expression consacrée. La conjecture technoscientifique repose sur un dispositif appelé «chambre de divertissements» (nursery) que les parents, George et Lydia, achètent pour que leurs enfants, Peter et Wendy, se tiennent tranquilles et qu'ils n'aient pas à s'en occuper. La "chambre" permet aux enfants de concrétiser leurs rêves et leurs pensées afin qu'ils puissent jouir des productions de leur propre imaginaire, s'y absorber à l'envie, préservés des turpitudes du monde extérieur. Bien vite, les parents s'aperçoivent que la chambre ne répond plus qu'à leur progéniture. Peter et Wendy ont matérialisé leur frustration et leur colère d'être ainsi délaissés sous la forme d'une savane africaine permanente, écrasée de soleil et grondante de bêtes sauvages. À terme, suite à une dispute, les enfants enferment leurs parents dans la "chambre» et assistent à leur dévoration par les fauves mécaniques. Les «notes» de Bradbury sur sa mise en scène exemplifient certaines des «techniques» recensées par Willingham: telle l'usage d'un "décor évocateur». Les préceptes de l'auteur de Fahrenheit 451 relèvent du «retour à Aristote» plébiscité par le critique américain :

Il fallait donc que le public devînt à la fois la savane et les lions au poil roussi par le soleil. Ainsi, quand ils se trouvaient dans la salle de jeux, mes acteurs promenaient leur regard sur l'auditoire transformé en immensité sauvage. Cette méthode donna d'excellents résultats. Nous dûmes également notre réussite au fait que nous utilisions du son diffusé des quatre coins de la salle grâce à des bandes magnétiques. Cela nous permit de faire rôder des lions rugissants autour du public 
laissant toujours celui-ci un peu sur le qui-vive, ne sachant jamais de quel côté allait s'élever des hautes herbes la rumeur des fauves ${ }^{25}$.

Selon cette perspective, le public tout entier est englobé, au propre et au figuré, dans la conséquence conjecturale de l'invention technique que représente la "chambre». L'œuvre de Bradbury mène une réflexion sur les rapports entre réalité et fiction et parvient à confronter les représentants de la société de consommation aux responsabilités éthiques qui leur incombent, et aux conséquences de leur dépendance au confort domestique. Le mélange d'effroi et d'étonnement que le sublime a pour ambition de causer chez le spectateur se trouve mis en abyme au travers de l'ultime attitude des enfants, Peter et Wendy, impuissants et déboussolés face au crime qu'ils ont perpétré. La pièce se referme avec eux, assis au bord du plateau, face public (ou face à la savane), les yeux ronds, le visage figé dans une expression horrifiée, incapables de prononcer un mot «comme s'ils avaient déjà éprouvé tout ce que la vie pouvait leur réserver, comme $s^{\prime}$ ils étaient au-delà de toute parole ${ }^{26}$. Le spectacle se termine ainsi. Les causes multiples du drame sont laissées à l'appréciation des spectateurs.

\section{Semper exploro}

La devise latine figure au brassard de tous les officiers de Starfleet, la ligue d'exploration spatiale de la saga Star Trek. Elle pourrait, à titre de clin d'oeil intertextuel, servir de conclusion à ces pages. La science-fiction a assurément beaucoup à apporter à la pensée du théâtre, mais l'horizon reste en friche, ouvert aux chercheurs en sciences humaines. Lambition de cette courte étude consistait surtout à démontrer la possibilité d'une telle investigation, à convaincre des liens fondés, de l'existence d'un espace fédérateur, entre philosophie de la scène et poétique science-fictionnelle. Mon propos espère avoir pu démontrer, d'une part, la nécessité de prendre en compte les procédés scéniques et enjeux pragmatiques du drame dans le tissage d'un tel questionnement commun. Cette contribution espère avoir d'autre part indiqué que le problème du sublime et les enjeux de ses formes contemporaines en esthétique pouvaient fournir, à cet égard, une

25. R. Bradbury, Introduction et notes sur la mise en scène, p. 20.

26. R. Bradbury, La Savane, p. 152. 
ligne de prolongation féconde. Il appartient aux études théâtrales comme à la philosophie de pousser plus avant les frontières de ces rapports, de progresser à travers les constellations de questions que ceux-ci ne cessent d'adresser aux penseurs, aux critiques et aux spectateurs.

Colin Pahlisch Université de Lausanne 


\section{BIBLIOGRAPHIE}

Atallah, Marc, L'art de la science-fiction, Yverdon-les-Bains, ActuSF et Maison d'Ailleurs, 2015.

Bouchard, Guy, "Science-fiction, utopie et philosophie: l'art de s'étonner", in Gilbert Hottois (dir.), Science-fiction et philosophie, Paris, Vrin, 2000, p. 43-66.

Bradbury, Ray, Théatre pour demain et... après, trad. par Jacques Legris, Paris, Denoël, 1973 [1972].

BReCht, Bertolt, Écrits sur le théâtre I, Paris, L’Arche, 1963.

—, Petit organon pour le théâtre, Paris, L'Arche, 2010 [1963].

Burke, Edmund, Recherche philosophique sur l'origine de nos idées du sublime et du beau, trad. par E. Lagentie de Lavaïsse, Paris, Vrin, 1973 [1757].

Hоттогs, Gilbert (éd.), Science-fiction et philosophie, Paris, Vrin, 2000.

Lardreau, Guy, Fictions philosophiques et science-fiction: récréation philosophique, Arles, Actes Sud, 1988.

Suvin, Darko, Pour une poétique de la science-fiction. Études en théorie et en histoire d'un genre littéraire, Montréal, Les Presses de l'Université du Québec, 1977.

Touraine, Alain, "La société comme utopie», in Lyman Tower (dir.), Utopie, la quête de la société idéale en Occident, Paris, Bibliothèque nationale de France, 2000, p. 28-38.

Willingham, Ralph, Science Fiction and the Theatre, Westport CT, Greenwood Press, 1994. 
